\title{
Textualisation et (re)conditionnement énonciatif
}

\author{
Catherine Détrie \\ CNRS - Université Montpellier 3, Praxiling UMR 5267 \\ catherine.detrie@univ-montp3.fr
}

\begin{abstract}
Dans cette contribution, je m'intéresserai à la façon dont l'interaction entre le scripteur et le lecteur s'inscrit en acte énonciatif, acte les engageant mutuellement en tant que coénonciateurs ${ }^{1}$, et se réalisant selon tel ou tel mode de textualisation, c'est-à-dire, très grossièrement, la façon dont la relation intersubjective entre les coénonciateurs s'inscrit dans le format d'énonciation du texte (cette notion est reprise et définie plus précisément ci-dessous). Je réfléchirai donc essentiellement à la textualisation (c'est-à-dire au processus de production de la matérialité textuelle nommée texte ${ }^{2}$ ), et au (re)conditionnement énonciatif que cette textualisation implique. Par ailleurs le texte, en tant que matérialité s'appréhendant par sa textualité (c'est-à-dire par son organisation textuelle globale, résultant d'une textualisation spécifique, qui permet de recevoir le discours comme un texte), est pris dans une dynamique intersubjective pratique, celle des coénonciateurs, dont on ne perçoit que le produit, l'objettexte. Mais en amont de cet objet-texte, il y a une intentionnalité qui le pense comme texte pour autrui, et donc une relation intersubjective fondatrice qui prédétermine la façon dont l'objet du discours va être représenté, et mis en forme linguistiquement.
\end{abstract}

Pour montrer l'effet de l'interaction enchâssante entre le scripteur et le lecteur dans la textualisation, je m'intéresserai essentiellement à des phénomènes langagiers dont la production de sens permet d'infléchir le mode de textualisation, et donc la textualité. Dans un premier temps, je mettrai en place le cadre de mon analyse, en proposant une définition de ces notions (1), avant de tenter, dans un deuxième temps, de les mettre en œuvre sur quelques processus discursifs qui ont pour effet d'opacifier le conditionnement énonciatif du texte, et de rendre de la sorte l'énonciation et la textualisation problématiques (2).

\section{De l'actualisation textuelle aux modes de textualisation : quelques repères}

Je m'appuierai sur la notion d'actualisation, empruntée à Bally (1932/1965), et Guillaume (1929/1970), que la linguistique praxématique (Lafont 1978) a retravaillée, et étendue, au-delà de l'actualisation nominale et verbale, à la phrase et au texte (Barbéris, Bres et Siblot 1998). Ainsi, outre la modélisation des règles d'organisation des séquences textuelles, et la gestion thématique, l'actualisation textuelle est conçue, par la linguistique praxématique, comme une opération graduelle, réalisant une explicitation / objectivation plus ou moins achevée de la mise en spectacle linguistique, c'est-à-dire «l'ensemble des opérations linguistiques par lesquelles le réel est représenté », "le spectacle linguistique se substitu[ant] ainsi au réel, le masqu[ant] et le manqu[ant], tout en posant son existence $»^{3}$. Cette réflexion a été amorcée à partir de la relecture critique des travaux de Benveniste (1958/1966) sur la subjectivité dans le langage, fondée sur un ego, être de parole inscrit dans l'activité d'énonciation. Dans le cadre de la notion d'actualisation textuelle, la praxématique propose ainsi une modélisation des formes de subjectivité qui tente de rendre compte de la variété des fonctionnements linguistiques, et qui distingue la subjectivité en même et en soi-même. Elle est ainsi appelée à substituer au terme d'énonciation celui de coénonciation, le sujet étant constamment pris dans son interaction avec un autre. Dans ce cadre, le sujet-ego est un point d'aboutissement du système d'actualisation: la réalité s'organise autour d'un centre explicite - une ipséité affirmée, construite. Barbéris (1998) montre ainsi que le sujet constitué (ego) n'est que l'étape finale d'un processus de construction de la subjectivité, qui peut être saisie à divers moments de cette construction. La notion d'actualisation textuelle permet ainsi d'articuler la façon dont se construit, dans sa dimension textuelle, la subjectivité (qu'on peut saisir à des degrés divers), la vision orientée, sur la base de l'expérience sensible, que le texte institue, et le format d'énonciation qu'il met en place. 
Cette modélisation de la subjectivité peut être saisie à partir de deux étapes prépondérantes, mais qui doivent être envisagées de manière graduelle. La première propose un type d'actualisation du texte antérieur à l'atteinte de la pleine objectivation, se dédoublant en deux positions de subjectivité : l'une, liminaire, est fondée sur un non-dégagement de la subjectivité individuelle - un même de similitude, où le sujet ne se représente pas réflexivement comme un être spécifique, différent de tous ses semblables, et dont les principaux marqueurs linguistiques sont les modes non personnels et l'absence de déterminant; l'autre, émergente, se manifestant par un dégagement imparfait du sujet - un même analogique, où le sujet n'opère pas de disjonction des points de vue ${ }^{4}$, autrui étant envisagé comme un même. La seconde étape - celle de la subjectivité en soi-même - correspond au mode discrétisé du je-tu-ici-maintenant se posant comme repère-origine à la mise en spectacle temporelle (il définit le maintenant) et spatiale (il est le repère de l'ici), et posant autrui comme autre. L'actualisation est pleinement réalisée, le spectacle linguistique se présentant comme ayant atteint le seuil d'objectivité. Ces marques de subjectivité, couplées avec les degrés d'objectivation des autres éléments du message, permettent de dégager deux types de construction subjective, qui donnent lieu à deux types généraux de textualité - la textualité en soi-même et la textualité en même-, pensés comme les « deux pôles extrêmes (implicite vs explicite), entre lesquels existent des types intermédiaires » (Barbéris, in Détrie, C., Siblot, P., Verine, B. éd., 2001 : 354).

Par la suite, Détrie et Verine (2003) ont repris cette réflexion, et proposé de remplacer textualité en même / soi-même par mode de textualisation en même / soi-même, ayant remarqué que :

\begin{abstract}
le mode de représentation du spectacle linguistique construit par un texte ne se confond ni avec la présence ni avec la somme des marques linguistiques de la subjectivité en même ou en soi-même, mais il naît des rapports spécifiques que la combinatoire textuelle établit entre ces marques. [...] Nous préférons [donc] aujourd'hui parler du mode de textualisation [...], que nous définirons comme la combinaison des choix formels permettant de décrire sa profondeur énonciative et la plasticité de l'intersubjectivité qu'elle construit: fondé sur les variations de positionnement de la subjectivité énonciative et les degrés d'actualisation de l'espacetemps (textualité en même vs en soi-même au sens strict), le mode de textualisation inclut le cas échéant l'hétérogénéité ou l'hybridité typologique, les degrés d'explicitation des relations intra- ou interphrastiques, le réglage axiologique ou tropologique du vocabulaire. $(2003: 215)$
\end{abstract}

Il convient maintenant de préciser que les positionnements en même ou en soi-même travaillent l'émergence d'un ethos différent, le premier s'appuyant sur des processus identificatoires, voire la fusion des points de vue, l'indifférenciation subjective (cas en particulier de la subjectivité en même de similitude, sous-tendue par une relation empathique), alors que l'ethos construit par un mode de textualisation en soi-même s'élabore à partir de positions discrétisées, et de l'objectivation des coénonciateurs. Lorsque le mode de textualisation en même est dominant, l'énonciateur envisage son rapport à l'énonciataire de manière non disjonctive, et la relation intersubjective construite implique leur indissociabilité et leur co-orientation. Ces phénomènes peuvent être repérés à des marqueurs aussi différents que l'énallage de la personne (Détrie 2008), les apostrophes en mon pauvre qui peuvent marquer un rapport d'empathie (Détrie 2006: 182-186), le choix d'outils prépersonnels comme le pronom on (Détrie 1998) ou les formes de l'infinitif. Ces marqueurs, immédiatement repérables, signalent souvent, au sein de l'énoncé, un repositionnement énonciatif du locuteur / scripteur.

Par contre, le mode de textualisation en soi-même correspond à un egocentrage affirmé, qui pose en discrimination radicale (en autre du je) le coénonciateur, et tout ce qui représente l'ailleurs de la relation interpersonnelle, discriminé en non-personne. On obtient ainsi deux configurations :

- la première positionne les actants narratifs comme dans une bulle énonciative non reliée à l'icimaintenant du scripteur. Cette dernière est quasiment la norme des textes littéraires. En effet, conventionnellement, la tradition romanesque présente le lecteur comme un tiers, l'interaction entre le scripteur et le lecteur, mais aussi entre le narrateur et le narrataire étant une interaction in absentia. Le spectacle, dans ce cas ne met pas en avant l'egocentrage, mais, s'appuyant sur cet egocentrage (la discrimination entre personne et non-personne, et au sein de la personne, entre $j e$ et $t u$ ), pose en pleine 
objectivité le spectacle romanesque, par le biais d'une narration en non-personne, soit un mode de textualisation en soi-même, sur le mode de l'objectivation de soi, ce qui permet de représenter l'ailleurs, le narré en pure extériorité objective. C'est en particulier le cas pour la plupart des romans du XIXe (parangon du narratif), qui posent l'instance textuelle en discrimination radicale à la fois du scripteur et des personnages, et mettent en scène une communication différée, le destinataire du roman n'étant pas posé comme partie prenante de la situation d'énonciation. Il n'y a donc aucun marqueur d'identification à l'un ou l'autre des pôles énonciatifs.

- La seconde configuration explicite l'inscription du je et du $t u$, l'interaction coénonciative entre le narrateur et le narrataire est alors exhibée. C'est le cas pour les textes de Diderot et Marivaux présentés en (2.1). Il en va de même pour Un dimanche au cachot, roman de P. Chamoiseau (2.2) : l'héroïne est en non-personne, tandis que le narrateur en je joue avec les indices biographiques du scripteur pour mieux égarer le lecteur sur la fausse piste d'un texte autobiographique (configuration la plus fréquente des romans de Chamoiseau).

Par contre, dans L'Esclave vieil homme et le molosse (2.2), l'inscription du narrateur dans le dire est beaucoup plus ténue, sous forme de traces, mais le roman présente un système d'énonciation tout à fait original, puisque la narration - dont l'objet est le cheminement, jusqu'à son accomplissement en destin, du personnage principal, un vieil esclave qui a décidé, contre toute attente, de fuir la plantations'effectue d'abord en non-personne, puis bascule à un moment donné du côté du je. Ce basculement ne modifie pas le mode de textualisation, qui reste un mode en soi-même, mais remanie le point de vue énonciativo-narratif.

La notion de mode de textualisation présente ainsi l'intérêt de mettre en perspective les phénomènes d'identification, de synchronisation coénonciative, ou au contraire de dissensus. En effet, parce qu'il construit un point de vue sur le monde, le mode de textualisation en propose une version, qui peut être celle d'une objectivation référentielle, ou au contraire celle d'une coconstruction, à partir d'une indifférenciation subjective, et qui exige du lecteur sa participation active. Pour montrer la variété et la gradualité de ces fonctionnements, je m'intéresserai à quelques outils de reconditionnement énonciatif, dont la mise en œuvre opacifie, sur le plan énonciatif, des textes qui, par ailleurs, s'appuient globalement sur un mode de textualisation en soi-même. Ces outils vont poser la disjonction personnelle comme problématique :

- soit parce qu'elle est instrumentalisée à des fins de brouillage des paliers énonciatifs : c'est le rôle de l'apostrophe lecteur dans Jacques le Fataliste, à la fois outil de textualisation en soi-même, mais aussi de reconditionnement global de l'entier textuel, visant à impliquer directement le lecteur réel dans l'acte narratif, et donc à proposer la représentation d'un texte coconstruit ;

- soit parce que le texte (Le Spectateur français de Marivaux) raffine le feuilletage énonciatif au point de dérégler la textualisation en soi-même, par la prolifération des instances subjectives en je, prolifération qui a pour effet non d'appuyer la disjonction personnelle que mettent en place habituellement les énoncés égocentrés, mais au contraire de la biaiser ;

- soit enfin parce que le texte intègre, au sein d'une textualisation en soi-même, des outils dont la fonction est de travailler l'indifférenciation subjective, et donc le mode de textualisation en même. C'est le rôle en particulier de l'infinitif en emploi autonome ou de la phrase nominale dans l'œuvre de P. Chamoiseau. 


\title{
2 D'un rôle discursif à un rôle énonciativo-textuel : quelques outils de (re)conditionnement énonciatif
}

\author{
De certains effets de sens inattendus de la coénonciation montrée
}

\section{Le (re)conditionnement énonciatif opéré par l'apostrophe}

Une des fonctions de l'apostrophe, dans le rapport à autrui, est d'instaurer un axe frontal, de face à face. Fondant la relation coénonciative, l'apostrophe constitue donc un processus d'assertion forte du mode de textualisation en soi-même, soit un mode permettant d'opposer les coénonciateurs au sein du couple je / $t u$, ce qui correspond à l'explicitation de l'énonciataire. Elle s'avère à ce titre un outil de la construction textuelle de la subjectivité en soi-même. Elle en est un des marqueurs privilégiés, puisque son rôle est de matérialiser la relation à la deuxième personne : le degré d'objectivation d'autrui est alors maximal. L'apostrophe met ainsi en spectacle la rupture, la différence, l'altérité, et donc la reconnaissance d'autrui comme autre du je. L'intersubjectivité est construite sur le mode de l'individuation subjective, c'est-à-dire d'une relation à l'autre basée sur son irréductible identité individuée, et non sur une consensualité qui ne permettrait pas la discrimination indubitable de $j e$ et $t u$. L'apostrophe joue de la sorte un rôle important dans la construction textuelle de la subjectivité, puisqu'elle envisage la relation coénonciative sur le mode disjonctif, où tout ce qui n'est pas je est pensé comme autre (cf. Détrie 2006).

Ce faisant, la mise en scène de la coénonciation, construite par l'apostrophe, signale une attitude énonciative spécifique de l'énonciateur, et influe sur la lecture / compréhension / interprétation globale du texte en question. Par voie de conséquence, l'apostrophe peut être au fondement d'une lecture adressée pour un texte dans sa totalité, alors que ce texte est par ailleurs construit en désengagement de la personne. Elle met alors en place une relation interpersonnelle forte, en affichant de manière ostentatoire la discrimination intersubjective. En effet, le fait que l'apostrophe nominale se règle par une lecture déictisante a des conséquences non seulement sur le cotexte étroit (elle confère une orientation allocutive à la phrase), mais aussi sur le cotexte large (elle transforme automatiquement le discours qui l'accueille en discours adressé), sur l'entier du discours et sur la textualité elle-même. Elle s'avère de la sorte un élément structurant de la totalité textuelle, et modifie en conséquence la production de sens de l'entier du texte, en mettant en spectacle l'interaction entre le narrateur et le narrataire : le positionnement du narrataire en coénonciateur ratifié travaille non seulement la dimension coénonciative de l'énoncé, mais aussi la dimension textuelle, et façonne tel ou tel type de textualité.

Avant d'aborder le rôle de l'apostrophe dans Jacques le fataliste, il me paraît nécessaire de rappeler que, pour une œuvre littéraire, la scène englobante ${ }^{5}$ - celle qui s'instaure entre le scripteur et le lecteur - est généralement implicitée : si l'interaction entre le scripteur et le lecteur est indubitable, elle est néanmoins in absentia, dans la mesure où le scripteur cède la place à un narrateur, cette hiérarchisation étant définitoire du roman : aussi ne poserai-je pas en discrimination, dans le choix de la symbolisation $E_{l}$ (l'énonciateur enchâssant) le narrateur et le scripteur, ni dans celle de $E_{2}$ (l'énonciataire enchâssant) le lecteur et le narrataire. La présence du scripteur ou du lecteur résulte d'un effet de sens de la narration elle-même. Cet effet de sens est cependant capital, puisqu'il induit le traitement de l'interaction narrateur / narrataire au palier de l'interaction scripteur / lecteur, le but étant que le lecteur réel interprète le texte à l'aune de cette dernière. Au fil de l'analyse, je préciserai simplement, en termes d'effet de sens, la représentation privilégiée, du narrateur ou du scripteur, du narrataire ou du lecteur.

Ce jeu est exemplaire dans le texte Jacques le Fataliste de Diderot, texte romanesque atypique, mettant en scène de manière ostentatoire l'hétérogénéité énonciative, en récusation d'une énonciation se donnant comme homogène, et qui ne ferait entendre que la voix de l'instance narrative. En effet, cette illusoire homogénéité est battue en brèche par les 56 occurrences de lecteur en apostrophe, qui constituent énonciativement, de manière très concrète, puisqu'elles le corporalisent, un narrataire à qui est accordé le statut de lecteur: dès lors qu'il existe dans la matérialité textuelle, il peut être pris à partie par le narrateur, qui peut lui donner des ordres, le conseiller, lui suggérer telle ou telle piste interprétative, etc., 
si bien que l'énonciation adressée à $E_{2}$ peut être interprétée non seulement comme un dialogue, dans la fiction, entre le narrateur et le narrataire, en tant qu'image du lecteur, mais aussi comme un dialogue entre le scripteur et le lecteur réel, dont le texte cherche l'adhésion, construit un mode de lecture, en configurant un guidage pour l'interprétation globale, et en proposant une diégétisation «liée au mode énonciatif actualisé » (Adam, 2005 : 200). L'effet est de placer tout le texte sous la dépendance de cette interaction entre le scripteur et le lecteur, grâce à ces 56 occurrences de lecteur.

Par un tour de passe-passe énonciatif, en relation avec une scénographie inhabituelle, en déport du contrat générique, dans la mesure où elle construit un coénonciateur-lecteur de la narration en train de se faire, exhibant ainsi des figures de coénonciateurs, l'apostrophe lecteur engage une procédure de référenciation directe du coénonciateur, et lui accorde un statut en rapport avec l'acte de lecture lui-même du texte, donc dans une relation étroite à l'énonciateur envisagé dans son activité scripturale. Le narrataire-lecteur, apostrophé, est ainsi positionné en tant que coénonciateur de l'interaction entre le scripteur et le lecteur. L'apostrophe lecteur, qui se contente discursivement de matérialiser un poste énonciatif dans la narration (un lecteur fictionnel), est de la sorte envisagée par le lecteur réel comme une adresse directe à sa personne. Les deux niveaux de coénonciation, celui de la scène englobante et celui de la scénographie, se superposent alors constamment. Aussi, que ce lecteur soit réel ou fictif (être de chair ou figure de lecteur) importe peu. L'important est que la scénographie, qui produit et valide cette configuration particulière narrateur / narrataire, se construit à l'aune de la scène englobante, s'alignant sur cette dernière au plan énonciatif: dès lors le lecteur réel reçoit le texte comme un texte qui lui serait directement adressé et s'identifie à l'acteur diégétique qu'est le lecteur fictionnel interpellé, le procédé créant l'illusion d'une négociation interactionnelle de l'acte de narrer dont il serait un actant direct. En effet, les 56 occurrences de lecteur sont systématiquement en relation avec des procès perceptifs, métacognitifs, métadiscursifs ou métanarratifs. Plusieurs configurations peuvent être ainsi dégagées.

a. Incitation au partage expérientiel grâce à des procès perceptifs ou métacognitifs imputés à $\mathrm{E}_{2}$ :

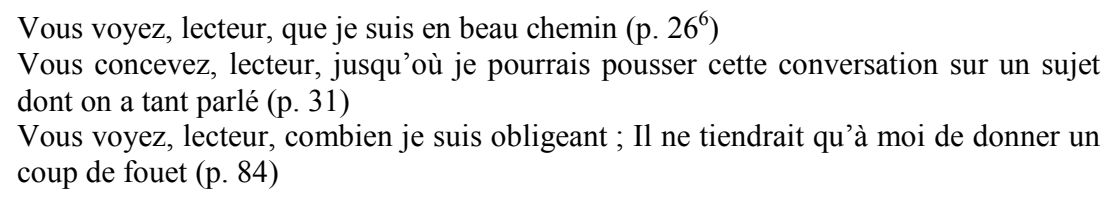

b. Mise en scène de l'activité interactionnelle elle-même.

Dans le cadre d'une pseudo-discussion avec son coénonciateur $E_{2}$, le narrateur $E_{1}$ commente leur comportement interactionnel, l'apostrophe appuyant des marqueurs métadiscursifs (dire), métadialogaux (interrompre, question, parler), des paroles narrativisées (éloges, blâmes), ou des procès propices au dialogue (écouter...) :

Mais si vous m'interrompez, lecteur, et si je m'interromps moi-même à tout coup, que deviendront les amours de Jacques? (p. 59-60)

$\mathrm{Ah}$ ! lecteur, la patience avec laquelle vous m'écoutez me prouve le peu d'intérêt que vous prenez à mes deux personnages, et je suis tenté de les laisser où ils sont... (p. 89) Et vous, lecteur, parlez sans dissimulation ; car, vous voyez que nous sommes en beau train de franchise (p. 138)

$\mathrm{Ah}$ ! lecteur, vous êtes bien léger dans vos éloges, et bien sévère dans votre blâme. (p. 186)

Je vous entends, lecteur : vous me dites : «Et les amours de Jacques ?... (p. 203)

Lecteur, tandis que ces bonnes gens dorment, $\mathrm{j}$ 'aurais une petite question à vous proposer à discuter sur votre oreiller (p. 219)

c. Suggestion de fausses pistes narratives aussitôt récusées :

Vous allez croire, lecteur, que ce cheval est celui qu'on a volé au maître de Jacques : et vous vous tromperez. (p. 61)

[...] je voudrais bien savoir ce que vous en penseriez ? Que je me suis fourré dans une impasse à la Voltaire, ou vulgairement dans un cul-de-sac, d'où je ne sais comment sortir, et que je me jette dans un conte fait à plaisir, pour gagner du temps et chercher 
quelque moyen de sortir de celui que j'ai commencé. Eh bien, lecteur, vous vous abusez de tout point. (p. 108)

d. Réparation de pseudo-oublis narratifs :

J'ai oublié de vous dire, lecteur, que Jacques n'allait jamais sans une gourde remplie du meilleur (p. 250)

e. Pointage de ses propres fantaisies et failles narratives :

Mais, lecteur, quel rapport cela a-t-il avec le voyage de Jacques le Fataliste et de son maître ?... (p. 60)

Il me prend envie, lecteur, de vous entretenir de la naissance de la dive Bacbuc (p. 252)

Lecteur, qui m'empêcherait de jeter ici le cocher, les chevaux, la voiture, les maîtres et les valets dans une fondrière? (p. 281)

f. Justification de ses choix narratifs ou de son rythme narratif :

Lecteur, si je faisais ici une pause, et que je reprisse l'histoire de l'homme à une seule chemise (p. 108)

Premièrement, lecteur, ce ne sont pas des contes, c'est une histoire, et je ne me sens pas plus coupable, et peut être moins, quand j'écris les sottises de Jacques, que Suétone quand il nus transmet les débauches de Tibère. (p. 248)

g. Justification ou revendication métadiscursive de ses propres choix lexicaux :

Hudson, impatienté de ces questions, et bien convaincu que Richard ne le prendrait pas pour un saint, lui dit brusquement : «Mon cher Richard, vous vous foutez de moi, et vous avez raison. "

Mon cher lecteur, pardonnez-moi la propriété de cette expression (p. 217)

Ah! hydrophobe ? Jacques a dit hydrophobe ?... Non, lecteur, non ; je confesse que le mot n'est pas de lui. (p. 297)

h. Construction d'un personnage à partir du portrait du lecteur, tel qu'il est aspectualisé par le narrateur :

\footnotetext{
Il était homme. - Homme passionné comme vous, lecteur; homme curieux comme vous, lecteur; homme questionneur comme vous, lecteur; homme importun comme vous, lecteur. - Et pourquoi questionnait-il ? - Belle question! Il questionnait pour apprendre et pour redire comme vous, lecteur... (p. 71)
}

Le fait que l'apostrophe soit chaque fois impliquée dans des procès d'ordre perceptif et narrativoénonciatif témoigne de son rôle d'organisateur énonciatif global, qui s'avère aussi un rôle fédérateur d'homogénéisation de l'hétérogénéité énonciative définitoire du roman, et par là même, un rôle, macrostructurel, de hiérarchisation textuelle. En affichant la coénonciation entre le narrateur et le narrataire, elle construit discursivement ce dernier comme un lecteur de la narration proposée : ainsi y a-til interaction entre le tout textuel et la sphère coénonciative enchâssante, c'est-à-dire, pour un texte écrit, celle du scripteur et du lecteur.

C'est l'apostrophe au lecteur, et souvent elle seule, qui permet cette construction, la rappelle stratégiquement, et implique directement $\mathrm{E}_{2}$, en tant que narrataire / lecteur réel dans la disposition narrative, en lui conférant une fonction dans le système narratif. Le texte acquiert ainsi une profondeur énonciative, le lecteur étant un élément constitutif de la textualité. L'apostrophe lecteur correspond alors non seulement à une opération discursive qui transforme " un usage descriptif en usage déictique » (Pop 2001 : 261), mais aussi à une opération textuelle proposant une lecture déictisante de l'entier textuel, le palier déictique modelant directement la textualité : il y a intrusion explicite de l'énonciation dans l'énoncé, ce qui a pour effet d'ouvrir un espace discursif (au sens de Pop, le détachement signalant un rehaussement, d'une lecture grammaticale à une lecture pragmatique, en relation directe avec le lecteur réel). Cette textualisation de l'apostrophe aboutit à la conversion d'une lecture seulement descriptive (le narré) en une lecture déictisante de l'entier textuel, superposant deux modes d'énonciation. 
En outre, la répétition lexicale (les 56 occurrences de lecteur) dessine une organisation textuelle globale qui lui confère une texture particulière, et cela tout à fait au-delà de l'organisation romanesque (constamment faite de lignes brisées, de fausses pistes, etc., mais qui propose un parcours grosso modo linéaire). Cette répétition lexicale est aussi une scansion de la configuration énonciativo-déictique (toutes les occurrences de lecteur sont en apostrophe), puisqu'elle raccroche la narration à une situation énonciative dont la figure du lecteur est partie prenante. À ce titre, l'apostrophe est non seulement un organisateur énonciatif, comme on l'a dit ci-dessus, mais aussi un organisateur de l'entier textuel, qui propose une textualisation fortement orientée vers autrui tout en signalant, de la part du scripteur, un positionnement en soi-même, et travaille l'émergence d'un ethos spécifique, à partir d'un egocentrage affirmé : elle pose en discrimination radicale (en autre du je) le coénonciateur. Ainsi ce positionnement en soi-même fortement montré et délibérément egocentré opère-t-il un reconditionnement global de l'entier textuel, puisque la totalité romanesque ne peut se lire qu'à l'aune de la relation construite entre $E_{1}$ et $E_{2}$, qui, habituellement, on l'a signalé plus haut, n'ont pas d'existence discursive, l'instance textuelle étant seulement positionnée en creux. On obtient ainsi un texte qui privilégie ostentatoirement un mode de textualisation en soi-même, tout en superposant les figures du scripteur et du narrateur, celles du narrataire et du lecteur, et en jouant d'une communication qui ne serait pas vraiment différée, contrairement à la plupart des autres types narratifs. Ici, au contraire, par cette mise en scène d'un dialogue, et surtout d'une coopération affichée entre les instances de la coénonciation, le récepteur se saisit de cette interaction posée comme la sienne, celle à laquelle il participe, la textualisation mettant en représentation un ton, un corps parlant, qui engendre l'identification du lecteur réel, la corporalisation du message étant une constante de cette textualisation en soi-même particulière.

\section{Un mode de textualisation en soi-même déréglé par le feuilletage énonciatif: l'exemple du Spectateur français de Marivaux}

Inversement, la disjonction des personnes, habituellement indice d'un mode en soi-même, quand elle donne lieu à une démultiplication des scènes d'énonciation passant par je/vous, peut aboutir à un brouillage des positions subjectives, et à une textualisation indécidable. C'est le cas par exemple des Journaux de Marivaux, et plus particulièrement du Spectateur français. En effet, la construction textuelle s'élabore à partir d'une parole en je, un je qui se positionne comme l'énonciateur $\mathrm{E}_{1}$ (et que le lecteur est invité à traiter comme s'il s'agissait du scripteur), mais qui n'a de cesse de donner la parole à une multiplicité d'autres $j e$, le plus souvent enchâssés en interne (jusqu'à sept paliers d'enchâssement), sans délégation énonciative clairement formulée. En effet, la textualité a pour caractéristique de ne jamais proposer de liage entre discours rapportant et discours rapporté. Le système de l'autonomie des deux discours prévaut ainsi constamment, l'insertion du dire d'autrui s'effectuant toujours a minima (absence de guillemets, ou d'italiques, et rareté des verbes de locution), les délégations de parole s'effectuant de manière délibérément brutale. Voici un exemple :

C'est un jeune seigneur espagnol qui parle.

Chacun croit les usages de son pays les meilleurs et les plus sensés. Il y avait déjà quelque temps que j'étais dans les Gaules, quand un Français que j'avais vu en Italie vint me voir. (p. 127)

Les enchâssements énonciatifs constants (discours rapportés, délégations de parole, mémoires intégrés, billets, lettres, etc.) constituent ainsi l'aspect le plus original de la textualisation marivaldienne. Cette spécificité énonciative - la prolifération des énoncés rapportés (e), dont la plupart sont eux-mêmes enchâssés ( $\left.\mathrm{e}^{\prime}\right)$ dans des discours déjà rapportés -, dégage une multiplicité d'énonciateurs $\left(\mathrm{e}_{1}, \mathrm{e}_{1}{ }_{1}\right)$, eux aussi en $j e$. Ces $j e$ ancrent dans leur dire des vous (énonciataires $\mathrm{e}_{2}, \mathrm{e}_{2}$, etc.), ces derniers pouvant représenter (c'est le cas le plus fréquent) dans le discours d'autrui le je spectateur-narrateur $\left(\mathrm{E}_{1}\right)$.

La neuvième feuille illustre exemplairement cette complexité. Le spectateur-narrateur $\mathrm{E}_{1} \mathrm{y}$ rapporte une lettre qu'une jeune demoiselle ( $e_{1}$ ) lui a fait parvenir (p. 154-159), soit une première délégation de parole (e). À l'intérieur de cette lettre, construisant deux énonciataires $\left(\mathrm{e}_{2}\right)$ distincts - d'une part le destinataire de la lettre, soit $\mathrm{E}_{1}$, posé en vous ( «la lettre que je vous écris »), d'autre part l'amant de $\mathrm{e}_{1}$, posé lui-même en $t u$ (« tu le sais », « dis-moi », « tu m’offris ta foi ») -, je (soit l'énonciatrice $\mathrm{e}_{1}$ ) cède la parole à la fille 
de l'amie de sa mère, parole (e') elle-même en je $\left(\mathrm{e}_{1}{ }_{1}\right)$, un je posant l'épistolière $\mathrm{e}_{1}$ en vous, c'est-à-dire en position d'allocutaire, soit $\mathrm{e}_{2}$ (《je ne puis vous en dire davantage », p. 156). À l'intérieur de e', apparaît un dernier décrochage énonciatif (e") en je, celui d'un jeune homme $\left(\mathrm{e}{ }^{\prime}{ }_{1}\right)$, dont $\mathrm{e}_{1}$ rapporte les propos (p. 157-158) : la parole du jeune homme constitue ainsi le quatrième palier énonciatif. La feuille se clôt par une reprise en main du spectateur-narrateur ( "J'interromps ici la suite de cette histoire », p. 159).

Ajoutons à cette complexité l'autoreprésentation fréquente de la parole du spectateur-narrateur lui-même (assertée ou virtuelle) : «Mais, disais-je en moi-même, de quel expédient de vanité peut se servir une femme laide... [...] non, ce n'est pas cela, me répondis-je » (p. 124-125), ou «Peu s'en fallait que je ne disse : Ils ont raison » (p. 126).

Enfin, la scène coénonciative englobante est aussi représentée, puisque le texte accorde une existence textuelle au lecteur réel, et ce, dès la clôture initiale du Spectateur français : «Lecteur, je ne veux point vous tromper, et je vous avertis d'avance que ce n'est point un auteur que vous allez lire ici» (p. 114 $)$. Le fait de se refuser le statut d'auteur manifeste, si besoin était, que c'est l'instance du scripteur, et non celle du spectateur-narrateur, qui parle ici. Cette ouverture explicite la relation entre Marivaux et le lecteur, lui confère une concrétude et donc une épaisseur, soulignant la réalité de la communication littéraire.

Ces enchâssements énonciatifs sont articulés au pacte de lecture, celui d'un je qui n'est pas un je autobiographique, mais plutôt une figure du sujet d'énonciation : un je anonyme en quelque sorte, mais qui permet une écriture individualisée. Son corollaire, le vous, est lui aussi polymorphe, pouvant représenter les divers destinataires des discours directs enchâssés («Je disais l'autre jour à un de mes amis $[\ldots]$ : Savez-vous bien ce que chez certaines gens signifient ces mots : ils courent après l'esprit ? », p. 146), les critiques ( «C'est pour une bonne œuvre que je vous sollicite», p. 143) les hommes riches, les Modernes («vous prétendez valoir », p. 146), le narrataire-lecteur lui-même (« Voulez-vous savoir ceux à qui d'entre les partis », p. 150), etc. Les énonciations gigognes multiplient ainsi ad libitum énonciateurs et énonciataires.

L'effet de sens de cette textualisation tout à fait originale (la convocation d'extériorités énonciatives nourrissant la parole du spectateur-narrateur, mais aussi la mettant en sourdine, pour mieux faire entendre le bruissement des voix autres par le biais de la multiplication des je) est de construire un ancrage subjectif relativement indifférencié : cette multiplication des je - plus ou moins dilués et instables - rend en effet les repères fluctuants, voire inexistants. La voix textuelle est alors seulement le porte-parole et l'orchestrateur de ce bruissement, cette activité d'orchestrateur participant d'un ancrage subjectif luimême indifférencié. Le mode de textualisation en soi-même est de la sorte fragilisé par l'instabilité subjective. Le feuilletage énonciatif ne construit pas une personnalisation, malgré l'omniprésence du pronom je, mais plutôt une démultiplication - voire une indifférenciation - des voix, que le spectateurnarrateur orchestre, et par là même met à distance. Le glissement constant d'une énonciation à l'autre, la démultiplication des délégations de parole minent l'actualisation en soi-même dont les je procèdent, élaborant un mode de subjectivité peu ipséifiant. Le mode de textualisation construit s'avère alors aux confins d'un mode en même analogique.

Ainsi ces deux textes, au plan énonciatif, proposent un ancrage subjectif fort, étant assumés par une instance textuelle en je, et affichant (exhibant dialogiquement) l'opposition et la rupture entre les instances, soit un mode de textualisation en soi-même. Néanmoins, l'orchestration de l'intersubjectivité, telle qu'elle est mise en œuvre dans ces deux textes, va à l'encontre des attendus liés à la discrimination des coénonciateurs. En effet, les choix effectués impliquent tout le système d'appréhension du texte, et jouent donc un rôle considérable dans la production textuelle du sens. Dans Jacques le fataliste, le texte non seulement s'appuie sur la disjonction des personnes, mais encore exhibe, par l'apostrophe, la corporalisation du message qu'il effectue, tout en invitant le lecteur à s'impliquer dans la narration, à être partie prenante de sa construction, par le biais de lacunes narratives délibérées et signalées de manière ostentatoire par le narrateur. Dans Le Spectateur français, la disjonction des personnes, assertée dès la clôture initiale du texte, est progressivement brouillée par la prolifération des instances personnelles, ce qui induit plutôt une lecture empathique. Dans les deux cas, la textualisation en soi-même est utilisée non 
à des fins d'explicitation de l'intersubjectivité, mais au contraire à des fins de brouillage : l'effet textuel est alors une réorientation discrète du mode de textualisation du côté du mode en même, par la mise en spectacle de la coconstruction narrative chez Diderot, grâce à la superposition des paliers énonciatifs induisant une lecture empathique, par la stylisation et la désindividualisation du spectateur-narrateur, voire la relativisation de sa fonction, générée par la prolifération de ces mêmes paliers, chez Marivaux. L'emploi de marqueurs explicites de coénonciation comme les pronoms personnels, et tout particulièrement je, ou l'apostrophe, qui sont autant d'outils de la textualisation en soi-même, œuvrant à une discrimination radicale des coénonciateurs, peut ainsi, dans la dimension textuelle globale, gauchir leur réglage pragmatico-énonciatif dans le cotexte phrastique.

D'autres morphèmes, ceux-là prototypiques d'un mode de textualisation en même, peuvent aussi venir perturber, voire biaiser la textualisation en soi-même, que le texte, globalement, met en place. C'est ce cas de figure que je développe ci-dessous, en m'intéressant à deux indices de textualisation en même: l'infinitif en emploi autonome et la phrase nominale. Ces schémas syntaxiques sont une constante de l'écriture de Patrick Chamoiseau. J'exemplifierai donc ma réflexion sur ces deux phénomènes en m'appuyant sur son œuvre.

\section{De quelques outils de positionnement énonciatif en même requérant l'engagement pratique du lecteur}

\section{L'infinitif en emploi autonome, un outil de (re)conditionnement énonciatif}

L'infinitif est un mode non personnel et non temporel : il se contente de donner du procès représenté par le verbe une image virtuelle, sans commencement ni fin : cette image de temps à peine ébauchée est à rapporter à la chronothèse in posse (représentation du temps en puissance) selon la théorie guillaumienne (1929/1970 : 29) : il s'agit d'une image-temps «que la pensée n'a aucunement réalisée, mais qu'elle est, néanmoins, en puissance de réaliser », c'est-à-dire seulement « du temps intérieur à l'image verbale », et indépendant de la durée de l'action, l'infinitif correspondant à une saisie précoce de l'image verbale. Cette approche est importante dans la mesure où l'infinitif efface l'actant à l'origine du procès : on a donc affaire à un procès présenté dans sa plus grande extension, sans bornage personnel, et sans ancrage temporel. Dès lors, le positionnement énonciatif peut difficilement être en soi-même, puisque la forme infinitive ne permet pas l'inscription dans un espace-temps identifiable, que ce dernier appréhendé comme un ici-maintenant par la textualisation de Jacques le Fataliste, ou un ailleurs-autrefois, comme le font la plupart des textes romanesques.

Patrick Chamoiseau, dont le questionnement romanesque tourne systématiquement autour de la thématique de l'identité et de la mémoire, privilégie l'infinitif à de très nombreux moments de ce questionnement : l'infinitif permet en effet de surseoir à un conditionnement énonciatif incarné, de construire un no man's land énonciatif, en effacement des marques déictiques et temporelles, et par là même de proposer une image de l'instance textuelle non expressément discriminée, ce qui bloque l'émergence d'un actant éventuel. L'infinitif joue de la désinscription de l'ego énonceur, en position d'in posse sur le plan de l'actualisation subjective, inapte à s'inscrire précisément dans la réalité spatiotemporelle. Temps suspendu engendré par l'infinitif et subjectivité implicite (requérant l'investissement subjectif du lecteur) impliquent alors un recentrement subjectif sur le mode du co-partage avec le lecteur, posé en même. Voici un exemple de cet emploi, emprunté à L'Esclave vieil homme et le molosse, dont j'ai spécifié ci-dessus l'originalité sur le plan énonciatif. Cet extrait se situe après le basculement du texte en $j e$. L'esclave se trouve face à face avec un dangereux serpent, dont la piqûre est mortelle :

Maintenant mon sang retrouvait une panique. Une suée me vernissait le front. La terreur nous soudait dans un vrac silencieux. Nos effluves identiques trouvaient un équilibre. Cela sans doute me protégeait. Il fallait demeurer ainsi. Pas bouger, mon nègre. Pas foubin, mon bougre. Pas laisser ton cœur désaccorder sa peur. Pas laisser le monstre arrivant m'arracher une tremblade. Rester-là, avec cette Innommable plus puissante et plus rapide que toi. (p. $86^{8}$ ) 
Le vieil homme, dans ce moment de panique, est représenté comme éprouvant le besoin d'objectiver son corps en le dissociant de la personne subjective. Les indices de décorporalisation sont nombreux: expression de la sensation pure posée en désolidarisation de ce corps, comme si elle n'était pas secrétée par ce corps, mais qu'elle s'avérait un élément venu de l'extérieur (une suée), forme unipersonnelle (il fallait), enfin choix de l'infinitif: de l'autre du je $(t u)$ à l'indifférenciation personnelle opérée par l'infinitif (demeurer, pas bouger), dont on se rend compte ici qu'il correspond bien à un stade prépersonnel, c'est-à-dire à un stade où le dégagement de la personne n'est pas effectué, mais où la forme verbale porte en elle la programmation de la personne. L'hésitation entre une deuxième personne marquée par les deux apostrophes (mon nègre, mon bougre) ou la forme tonique du pronom (toi) et la première personne $\left(m^{\prime}\right)$ se résout sur le plan du procès par l'utilisation de l'infinitif: pas bouger, pas laisser le monstre arrivant arracher une tremblade.

Voici un deuxième exemple, emprunté à Un dimanche au cachot :

Tout se vole, tout se garde, tout peut servir. La ligne plonge. Vols contre vols. Apprendre à garder ses affaires. Apprendre à cacher. Calculer. Nettoyer, veiller à ses haillons. Se garder droit pour ne pas commencer à crever. Ne pas regarder ceux qui passent et qui voient sans te voir. La haine épuise. La peur éreinte. Cœur amer c'est fatigue, ceux qui l'ont crèvent tout de suite. Pas de haine, pas de peur, pas de cœur, oublier ça pour supporter ça. (p. $131^{9}$ )

On remarquera que l'infinitif est articulé à d'autres phénomènes de textualisation en même: article zéro pour certains syntagmes nominaux (Cœur amer c'est fatigue), transitivité absolue pour les verbes épuiser et éreinter (La haine épuise / La peur éreinte) qui permet de laisser dans le flou celui qui éprouve le sentiment en question, genre non marqué, qui signale la saisie indifférenciée, pour l'adjectif droit, alors que le personnage est féminin, choix de morphèmes sémantiquement non restreints (ça, tout) : autant d'outils permettant une lecture empathique. En particulier, ça signale, en creux, un foyer de perception (un sujet scopique), source du point de vue, sa valeur déictique (ça procède de la référenciation exophorique, et désigne directement une réalité du monde dans l'environnement immédiat du locuteur) instaurant un effet de mise en présence, tout en invitant le lecteur à partager l'expérience perceptuelle de ce sujet scopique. L'expérience étant posée comme partagée, il s'avère inutile de nommer plus précisément ce que recouvre ça. Ces morphèmes, construisant une subjectivité diffuse, signent tous, à ce moment du texte, un basculement de la textualisation du côté du même, sur la base d'un ancrage subjectif délibérément indifférencié.

Ainsi toutes ces phrases dont l'infinitif est le noyau sont-elles très faiblement actualisées, et signalentelles un engagement peu marqué qui privilégie l'indétermination sur le plan de l'actantialité : le texte construit ici un centre d'expérience à la source des procès à l'infinitif en deçà de positions subjectives discriminées, qui, cependant, permet l'adhésion immédiate du lecteur, précisément parce que les positions énonciatives ne sont pas préréglées, mais se donnent plutôt à lire comme un canevas énonciatif élémentaire, accueillant par son indétermination même, au sein duquel la place de l'énonciataire est esquissée en même. On notera d'ailleurs le flou personnel en relation avec ces infinitifs, le texte passant d'une non-personne à la deuxième personne : apprendre à garder ses affaires, veiller à ses haillons, se garder droit / ne pas regarder ceux qui passent et qui voient sans te voir. Ce dernier pronom (te) permet d'interpréter a posteriori garder ses affaires, veiller à ses haillons, se garder droit comme des formes antérieures à la discrimination personnelle en première ou deuxième personne : garder mes/tes affaires, veiller à mes/tes haillons, me/te garder droit.

Même chose pour les infinitifs suivants, qui succèdent à deux phrases verbales dont les sujets sont des syntagmes nominaux déterminés par un article défini, qui n'est anaphorique de rien, les syntagmes n'ayant pas été présentés précédemment ou déterminés par une expansion qui permettrait une actualisation plénière (le corps, le muscle: de qui ?), et une subordonnée dont le sujet est le pronom prépersonnel on, marqueur d'une textualisation en même :

Même le corps blessé qui va crever demain veille à cicatriser. Même le muscle brûlant peut se calmer si on oublie demain... Demain, c'est un couillon. Il conte des contes aux ababas. Envoyer demain se promener, prendre aujourd'hui là même ce qu'il y a à 
prendre comme pour toute l'année! Couper la canne. Tourner. Dépailler. Déposer. Couper. Tourner... Éclater dans ces petits morceaux, et se tenir comme ça sans trop se rassembler ni calculer demain. (p. 131-132)

Dans une recette de cuisine, l'infinitif (prendre trois blancs d'œuf, les monter en neige...) invite le lecteur à se positionner comme actant du procès (vous allez prendre trois blancs d'œuf et les monter en neige). Ici, la valeur est aussi celle d'une invitation à vivre de l'intérieur ces procès, comme ils sont perçus par les coupeurs de canne. Cette identification est rendue possible parce que l'infinitif n'est porteur ni d'une temporalité, ni d'une personne, et, à ce titre, propose un ancrage énonciatif qui ne discrimine pas l'instance textuelle de son autre, le narrataire / lecteur. Il est donc l'indice d'une construction intersubjective qui travaille son adhésion, récusant la discontinuité de la relation intersubjective le plus souvent construite par la textualité romanesque, et proposant des représentations impliquant le cophasage perceptif (oublier ça), actionnel (se garder droit, couper, tourner) et affectif (pas laisser ton cour désaccorder sa peur). Tout aussi intéressant est l'emploi concomitant du verbe éclater (qui gomme la disjonction personnelle - par le choix de l'infinitif -, mais aussi actantielle - par l'ellipse du factitif, dont l'effet est de renforcer le cophasage : il s'agit en fait de faire éclater la canne en petits morceaux pour en extraire le jus) et du déterminant démonstratif ces (dans ces petits morceaux, au lieu de en morceaux, plus attendu), qui sature exophoriquement les morceaux de canne comme si l'esclave et le lecteur étaient un unique foyer de perception, simultanément à l'origine du regard fouillant pour dénicher, à l'intérieur des morceaux de canne, le divin caillot de sucre, soit une spatialité en partage (il s'agit ici d'un petit chapardage à l'insu du maître : « toute grappille est divine, il faut en jouir tout de suite : une goyave, un ver de palmiste, un caillot de sucre » p. 131).

\section{Phrase nominale et textualisation en même}

La phrase nominale, par définition, propose « une syntaxe liminaire, potentielle », en effaçant « tout verbe susceptible de hiérarchiser les éléments du spectacle linguistique », et contraint de la sorte le lecteur "à inscrire son travail interprétatif dans les béances du temps signifiant » (Verine, 1998 : 39-40). Qu'elle soit monorème ou non, thématique ou rhématique, elle a généralement pour fonction de proposer un point de vue, posé comme délié d'un quelconque énonciateur, proposant une simple nomination, par exemple famines ou exils dans le passage ci-dessous, emprunté à L'Esclave vieil homme et le molosse, qui asserte l'existence d'un processus nominalisé (avoir faim, s'exiler) sans actant l'assumant ou le subissant explicitement, ce qui brouille la relation actantielle. De même, déportation peut construire un sens actif ou passif, pointant le résultat du procès accompli par le déporteur, ou celui qui est subi par le déporté. C'est au lecteur à accompagner le scripteur dans son dire, et à reconstituer les relations absentes. Le point de vue délibérément non rattaché à un actant identifié est lié à l'expérience douloureuse de l'esclavage, si douloureuse qu'il n'y a plus d'assomption possible d'un sujet, ce dernier ne pouvant se manifester qu'en creux du dit. La phrase nominale procède, comme l'infinitif noyau de phrase, à un effacement déictique et temporel, la seule actualisation proposée étant celle qui est propre à la détermination, mais ce système est lui-même remis en cause dès la troisième phrase. En effet, l'actualisation zéro est révélatrice d'une saisie non pas conceptuelle, comme on l'explique fréquemment, mais d'une saisie en indifférenciation subjective. Par ailleurs, la phrase nominale interdit toute possibilité de dégager un quelconque actant :

La dent des chaînes. Le rouache du fouet. La déchirée des cris. Morts explosives. Famines. Massacrantes fatigues. Exils. Déportation de peuples différents forcés de vivre ensemble sans les morales et les lois du Vieux-monde. (p. 20)

Par ailleurs la phrase nominale cultive le flou sur le plan de l'interprétation : la déchirée des cris peut être comprise comme le fait que les cris déchirent le silence, ou que les cris sont déchirants (réactivation du tour pousser des cris déchirants). Il en va de même pour morts explosives, où l'adjectif peut être pleinement prédicatif (les morts sont explosives), ou simplement descriptif des morts (d'explosives morts). Le refus de phrases verbales, qui permettraient d'élucider la production de sens, et de restreindre les possibles interprétatifs, préserve non seulement l'ambiguïté, mais aussi - surtout-la plasticité énonciative, chacun pouvant s'investir dans un énoncé qui n'impose pas de schéma intersubjectif réglé d'entrée de jeu. 
Autre exemple, emprunté aussi à L'Esclave vieil homme et le molosse, et qui rend compte d'un début de noyade :

\begin{abstract}
Mais la terre se déroba. Un manman-trou. Profond. Le vieil homme qui fut esclave s’y engloutit d'un coup.

Il était tombé dans une de ces vieilles sources qui nourrissaient les bois-profonds. Noyade. Une eau glacée-glacée. Il retrouva les cauchemars des cales négrières. Les abysses. La mer sans vent. Le sel. Les vagues. Grand-gueule des squales. L'eau. L'eau. Il allait se noyer au profond d'une source.

(...) Il voulut crier un à-moi de douleur. Tragédie d'oxygène. Il voulut roucler de plaisir pour tant de félicité dans l'effacement de ses souffrances. Il mourait. Finirbattre. Terre blanche. Boue chaude. (p. 79)
\end{abstract}

On remarquera que toutes ces phrases nominales sont centrées sur l'expression d'une sensation brute, mais celui qui est censé l'éprouver n'est pas explicitement mis en scène, il est au contraire momentanément effacé. En effet, le dire semble émaner d'une instance anonyme de perception, proposant un spectacle linguistique basé sur des schémas d'expérience qui impliquent l'énonciataire de manière non disjonctive et l'engagent au partage du point de vue ainsi construit : l'indistinction temporelle (la phrase nominale construit un temps suspendu), énonciativo-actantielle (elle implicite les relations énonciative et actantielle), syntaxique (elle opacifie les relations entre les constituants : la boue est chaude / il s'enfonce dans la/une boue chaude ?) impliquent de manière non disjonctive la subjectivité du lecteur, et ouvrent ainsi la voie à son adhésion ou à son empathie.

Qu'il s'agisse des infinitifs ou des phrases nominales, tous deux inaptes à être porteurs d'une temporalité et d'une inscription personnelle explicites, leur emploi respectif signe la désinscription du scripteur de son dire. En effet, dans le dernier exemple, le vieil homme est dans les phrases verbales discriminé en non-personne, c'est-à-dire en objectivation achevée, le scripteur opérant une mise en spectacle linguistique posée comme extérieure à sa relation avec le lecteur. Mais, dans les phrases nominales, ce conditionnement énonciatif s'efface au profit d'une mise en spectacle coconstruite, le lecteur étant invité à établir lui-même les relations temporelles, actantielles ou syntaxiques manquantes, voire à y rajouter les accentuations affectives qui peuvent s'imposer à lui, par exemple pour un manman trou, manman ayant à la fois une valeur intensive (un très grand trou, un trou terrifiant) et affective, liée à sa forme même. C'est à cette condition que le texte acquiert sa plénitude sémantique. Les coénonciateurs sont, dans ce mode de textualisation, engagés dans une relation dyadique privilégiant la synchronisation coénonciative.

\title{
En guise de conclusion, un retour au titre
}

Pourquoi avoir choisi Textualisation et (re)conditionnement énonciatif pour le titre de cette contribution ? L'appel à communication invitait à réfléchir en premier lieu à «la logique de la textualisation »: la textualisation, telle qu'elle est pensée par la praxématique, m'a permis d'articuler les « niveaux micro- et macro-textuels », puisque j'articule des phénomènes micro-textuels (syntaxiques et sémantiques) à l'énonciation et à la relation entre les coénonciateurs. Il invitait aussi à réfléchir au «(re)conditionnement énonciatif », formule qui m'a paru heureuse pour ce que je souhaitais démontrer: les jeux sur la construction textuelle de la subjectivité, et sur les points de vue qu'elle actualise, envisagés sur le mode de la gradience, du biaisage, voire de la modification de la textualisation initialement mise en place, que ces jeux impliquent, en engageant le lecteur dans un réseau empathique qui modifie son positionnement initial de tiers, tel qu'il est pensé dans la tradition romanesque. Les dérèglements de la textualisation qu'effectuent, dans les textes présentés ci-dessus, la prolifération des personnes, la confusion délibérée des paliers énonciatifs, certains emplois de l'infinitif ou de la phrase nominale exigent ainsi l'investissement subjectif de l'énonciataire-lecteur.

J'ai ainsi pris le syntagme conditionnement énonciatif au sens de régime énonciatif propre au texte, mais aussi d'action visant à mettre en condition, à influencer autrui, ici le coénonciateur. Le préfixe re- signale alors l'action consistant à faire changer d'état, et donc la transformation de la textualisation grâce à des constituants de rang micro-textuel, mais dont la portée est d'ordre macro-textuel, puisqu'ils modifient la relation énonciative enchâssante. Le fait de mettre le préfixe entre parenthèses signale, dans le cadre de 
cette réflexion, le biaisage toujours possible des positionnements énonciatifs, et donc le refus d'une subjectivité en tout ou rien, c'est-à-dire en même vs en soi-même (un conditionnement énonciatif unique), qui nierait les positionnements intermédiaires.

\section{Références bibliographiques}

Adam, J.-M. (2005). La Linguistique textuelle. Introduction à l'analyse textuelle des discours, Armand Colin : Paris.

Bally, C. (1932/1965). Linguistique générale et linguistique française, Francke : Bern.

Barbéris, J.-M. (1998). Pour un modèle de l'actualisation intégrateur du sujet. In Barbéris, J.-M., Bres, J. et Siblot, P. (éd.), De l'actualisation, CNRS Éditions : Paris, 239-261.

Barbéris, J.-M., Bres, J. et Siblot, P. (éd.) (1998). De l'actualisation, CNRS Éditions : Paris.

Benveniste, É. (1958/1966). De la subjectivité dans le langage. In Problèmes de linguistique générale I. Gallimard: Paris, 258-266.

Détrie, C. (1998). Entre altérité et ipséité : statut énonciatif de «on » dans Sylvie. L'Information grammaticale, 76, Société pour l'information grammaticale : Paris, 29-33.

Détrie, C. (2006). De la non-personne à la personne : l'apostrophe nominale, CNRS-Éditions : Paris.

Détrie, C. (2008). L'énallage : une opération de commutation grammaticale et/ou de disjonction énonciative ? In Rabatel A. (coord.), Langue française, Figures d'énonciateurs dans l'analyse pragma-énonciative des figures, Larousse : Paris.

Détrie, C., Siblot, P., Verine, B. (éd.) (2001). Termes et concepts pour l'analyse du discours: une approche praxématique, Champion : Paris.

Détrie, C., Verine B. (2003). Modes de textualisation et production du sens : l'exemple de «Complainte d'un autre dimanche » de Jules Laforgue. In Amossy R., Maingueneau D. (éd.), L'analyse du discours dans les études littéraires, Presses universitaires du Mirail : Toulouse, 213-225.

Guillaume, G. (1929/1970). Temps et verbe, Champion : Paris.

Lafont, R. (1978). Le Travail et la langue, Flammarion : Paris.

Maingueneau, D. (2002). Article Scène d'énonciation. In Charaudeau, P., Maingueneau, D. (éd.), 2002. Dictionnaire d'analyse du discours, 515-518, Seuil : Paris.

Pop, L. (2001). Le détachement dans le groupe nominal : l'hypothèse de la conversion pragmatique. In Amiot D., De Mulder W., Flaux N. (éd.), Le Syntagme nominal : syntaxe et sémantique, Artois Presses Université : Arras, 125136.

Rabatel, A. (1998). La Construction textuelle du point de vue, Delachaux et Niestlé : Lausanne-Paris.

Verine, B. (1998). Un exemple d'actualisation textuelle en idem : «Walcourt» de Paul Verlaine. L'Information grammaticale, 77, Société pour l'information grammaticale : Paris, 38-42.

\footnotetext{
${ }^{1}$ Pour la praxématique, l'interaction entre un énonciateur et un énonciataire est au fondement de l'activité de langage, si bien que toute énonciation peut être définie comme une coénonciation. Pour un texte écrit, les coénonciateurs sont, au palier de l'énonciation, le scripteur et le lecteur, au palier de l'énoncé, le narrateur et le narrataire. Dans les discours oraux, les rôles de locuteur et allocutaire permettent de construire une situation de coénonciation qui est aussi une colocution.

${ }^{2} \mathrm{Au}$ sens praxématique, cf. J.-M. Barbéris, entrée Texte / textualité in Détrie, C., Siblot, P., Verine, B. (éd.) : «Un texte est une suite d'énoncés oraux ou écrits posés par leur producteur - et destinés à être reconnus par leur(s) destinataire(s) - comme un ensemble cohérent progressant vers une fin et parvenant à constituer une complétude de sens ».

${ }^{3}$ C. Détrie, entrée Mise en spectacle, in Détrie, C., Siblot, P., Verine, B. (éd.).

${ }^{4}$ Point de vue est compris dans le sens de prise en charge énonciative des énoncés, cf. en particulier Rabatel (1998).
} 


\begin{abstract}
${ }^{5}$ Maingueneau (2002) part de l'idée que «tout discours prétend instituer la situation d'énonciation qui le rend pertinent». La scène d'énonciation ainsi construite discursivement se subdivise en une scène englobante, qui « assigne un statut pragmatique au type de discours dont relève un texte », ici approximativement, c'est celle de la relation scripteur / lecteur potentiel, dans le cadre pragmatique de l'écriture / lecture littéraire, en tant qu'institution sociale. La scène générique est définie par les genres du discours particuliers, ici le roman, en relation avec le contrat générique. La scénographie « est instituée par le texte lui-même », s'écartant du préconstruit générique.
\end{abstract}

${ }^{6}$ Pagination édition Garnier-Flammarion (1970).

${ }^{7}$ Pagination Classiques Garnier, édition Bordas (1988). In Journaux et æuvres diverses.

${ }^{8}$ Pagination édition Gallimard (1997).

${ }^{9}$ Pagination édition Gallimard (2007). 